\title{
A BEST DOWN OVERVIEW ON REMOTE SENSOR SYSTEMS AND ITS APPLICATIONS
}

\author{
S. Naga Mallik Raj ${ }^{1}$, S. Neeraja ${ }^{2}$, N. Thirupathi Rao ${ }^{1}$ and Debnath Bhattacharyya ${ }^{1}$ \\ ${ }^{1}$ Department of Computer Science and Engineering, \\ Vignan's Institute of Information Technology, Visakhapatnam, AP, India \\ ${ }^{2}$ Department of Computer Science and Engineering, \\ Pydah Engineering College, Visakhapatnam, AP, India \\ mallikblue@gmail.com, neerajasreerama@gmail.com, \\ nakkathiru@gmail.com, debnathb@gmail.com
}

\begin{abstract}
In the recent years, Remote Sensor Structures (WSNs) have broadened making thought from both the examination gathering and veritable customers. This paper explains the probability of sensor structures which has progressed and made helpful by the joining of little scale electro-mechanical systems advancement, remote exchanges and mechanized contraptions. In any case, the seeing tries and the potential sensor frameworks applications are reviewed, and a blueprint of areas influencing the system of sensor structures is given. Advancement in the progress of sensor, for instance, Miniaturized scale Electro Mechanical Frameworks (MEMS), remote exchanges, introduced structures, passed on orchestrating and remote sensor applications have contributed an expansive change in Remote Sensor System (WSN) starting late. It accessorizes and updates work execution both in the field of industry and our dependably life. Remote Sensor System has been everything seen as used as a touch of various locales, especially for affirmation and checking in agribusiness and living space seeing. Condition watching has changed into a basic field of control and accreditation, giving reliable structure and control correspondence with the physical world. The purpose for this paper is to discuss clearly the most related problems of WSNs, from the application, chart and change points of view. For drawing out a WSN, really we need to depict the most fitting advancement to be used and the correspondence traditions to be executed (topology, hail managing techniques, and so on.). These decisions rely on various portions, over all the application necessities.
\end{abstract}

Keywords - WSN, Protocols, MEMS, Layers

\section{INTRODUCTION}

Advances in remote correspondence and microelectronic devices, provoked the change of low-control sensors and the game plan of tremendous scale sensor frameworks. These applications yield tremendous volume of dynamic, topographically scattered and heterogeneous data[19][20]. This rough data, if viably separated and changed to usable information through data mining, can support robotized or human-started vital/key decision. In this way, it is fundamental to make strategies to burrow the sensor data for plans in order to settle on clever decisions quickly. In any case, the seeing tries and the potential sensor frameworks applications are reviewed, and a blueprint of areas

Received: March 25, 2019

Reviewed: June 5, 2019

Accepted: June 25, 2019

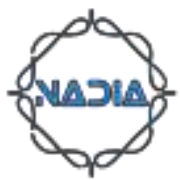


influencing the system of sensor structures is given. Advancement in the progress of sensor, for instance, Miniaturized scale Electro Mechanical Frameworks (MEMS), remote exchanges, introduced structures, passed on orchestrating and remote sensor applications have contributed an expansive change in Remote Sensor System (WSN) starting late [1]. It accessorizes and updates work execution both in the field of industry and our dependably life. Remote Sensor System has been everything seen as used as a touch of various locales, especially for affirmation and checking in agribusiness and living space seeing. Condition watching has changed into a basic field of control and accreditation, giving reliable structure and control correspondence with the physical world[15][16][17].

\section{ABOUT REMOTE SENSOR ARRANGE}

A Remote Sensor Organizer, is a type of inaccessible system that solidifies a wide range of spilling, self-composed, miniature, moo controlled gadgets named sensor centers called bits [21][22]. These systems without a question cover battery-worked presented contraptions that are composed to disliking of gathering, preparing, and exchanging of information to the managers, and have also controlled the limits of enlisting and the effort to be taken care of. The little PC's act like centers, which cooperate to outline the frameworks.

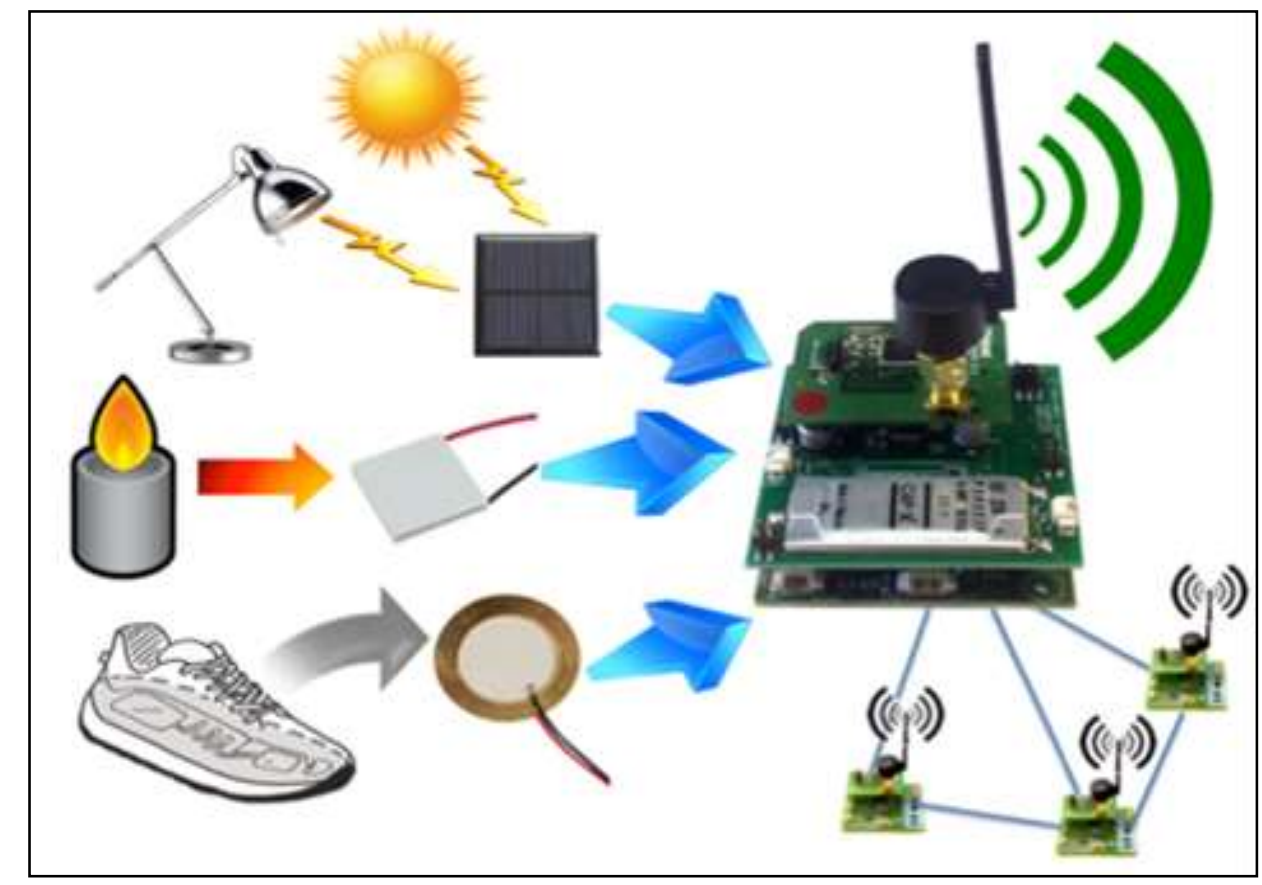

Fig. 1 Wireless Sensor Network [13]

[ Fig 1. Source:: https://www.elprocus.com/architecture-of-wireless-sensor-network-andapplications/ ]

The sensors center point is a multi-utilitarian, imperatively productive inaccessible contraption. A social event of sensors centers is to collect the data from the environment to achieve specific applications objectives. The correspondence among the bits should be grasped mentally for the utilization of handsets [2] [3][4][13]. In a sensor network, the amount of bits required can be hundreds or even thousands of autonomous microdevices (called as sensor nodes). Inquisitively with sensor networks, advertisement Hoc systems would be having lesser center focuses with no structure. 


\section{INACCESSIBLE SENSOR ARRANGE DESIGN}

The most broadly seen WSN building takes after the OSI arrangement. The layout of the WSN, wires 5 layers and 3 cross layers. For the most part in sensor networks we require 5 layers, specifically[13] Application layer, Transport layer, Network layer, Information interface or the Date Link layer and the Physical layer. The 3 cross planes are to be specifically- Control organization, Compactness organization, and Undertaking organization. They participate to raise the whole viability of the framework[23][24][25].

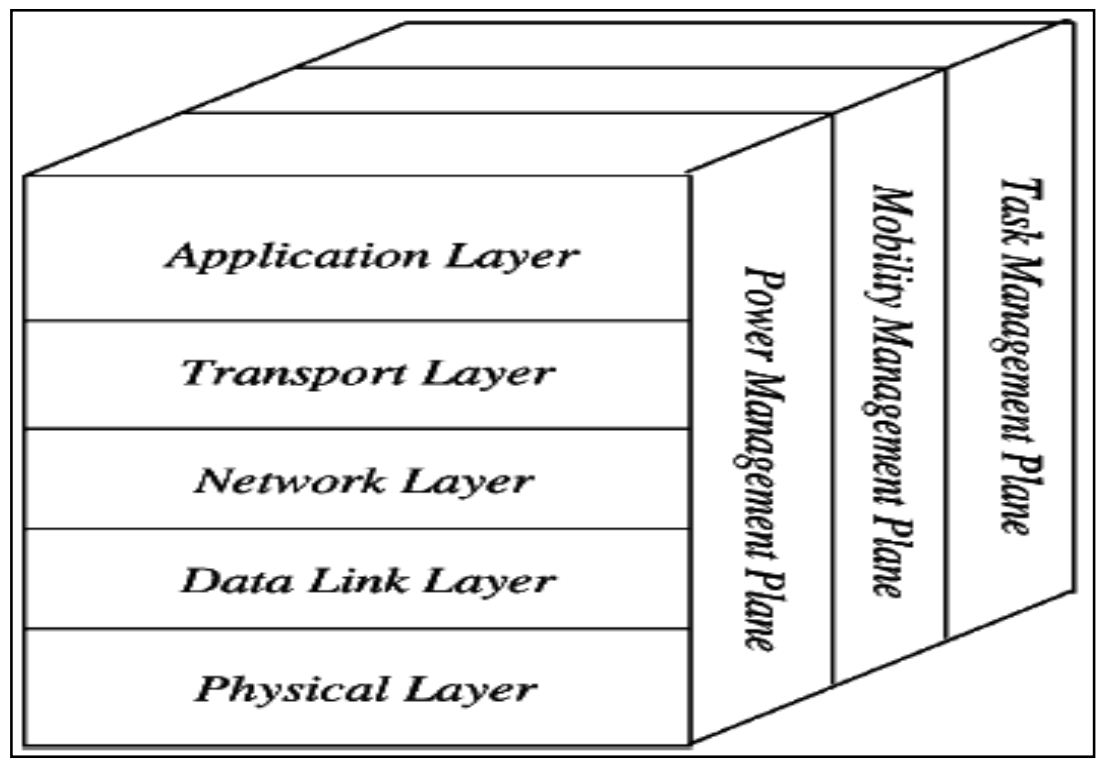

Fig. 2 WSN Architecture [11]

\section{$>$ APPLICATION LAYER}

The application layer is in hazard for improvement affiliation and provides programming for different applications that change over the data in a sensible shape to get the right information. Sensor structures have worked in different fields of applications, for instance, the commonplace, military, conditions, accommodations, etc. In any case, the seeing tries and the potential sensor frameworks applications are reviewed, and a blueprint of areas influencing the system of sensor structures is given. Advancement in the progress of sensor, for instance, Miniaturized scale Electro Mechanical Frameworks (MEMS), remote exchanges, introduced structures, passed on orchestrating and remote sensor applications have contributed an expansive change in Remote Sensor System (WSN) starting late. It accessorizes and updates work execution both in the field of industry and our dependably life. Remote Sensor System has been everything seen as used as a touch of various locales, especially for affirmation and checking in agribusiness and living space seeing. Condition watching has changed into a basic field of control and accreditation, giving reliable structure and control correspondence with the physical world[26][29].

\section{TRANSPORT LAYER}

The purpose of repression of the vehicle layer is to quit keeping up a vital separation and proceeding with quality, where a wide measure of traditions are anticipated that would offer this most remote point that are customary on the upstream. These traditions have striking structures for mishap affirmation and disaster restoration. This transport layer is absolutely needed when a structure is depended upon to approach contrasting frameworks. 
Giving a strong hardship recovery is a very noticeable imperative fit and that is the genuine reason why TCP is not fit for WSN. Transport layers can be withdrawn into Bundle and Occasion driven. There are a few unmistakable traditions in this layer, specifically STCP (Sensor Transmission Control Protocol), PORT (Cost Arranged Dependable Transport Convention) and PSFQ (Pump-Slowly, Fetch-Quickly).

\section{$>$ NETWORK LAYER}

The structure layer has an extraordinary measure of tries in setting of the application; everything considered, the essential errands are in the power sparing, midway memory and support. Sensors don't have a general ID and thus they must act like commonly made[27][28].

The basic idea of the sorting out tradition is to illuminate a strong way and the dull courses, as appeared by an incited scale called metric, which changes from custom to tradition. Among this are a huge measure of existing traditions for this framework layer, and they can be divided into- level controlling and hierarchal arranging or can be segregated into time driven, request driven and event driven[29][30].

\section{DATA LINK LAYER}

The Information interface layer is in hazard for multiplexing information plot run, data streams, Macintosh, and bungle control; insisting the continuing on nature of point-point (or) point-multipoint.

\section{$>$ PHYSICAL LAYER}

This layer gives a way for trading a surge of bits over a physical medium. It is fit for the decision of repetition, hail certification, change and data encryption. IEEE 802.15.4 is embraced as a standard for moo rate of a specific area and unavailable sensor that relate easily, control the use, thickness and the level of correspondence to refresh the battery life. CSMA/CA is used to offer assistance and shared topology. Presently a few changes has been made to IEEE 802.15.4.V.

\section{CONVENTIONS}

Here are a couple of traditions proposed for WSNs (Remote Sensor System). The Macintosh (Medium Access Control) layer reacts to this probabilistic get-together data by changing the measure of certifications to more retransmissions. It is seen as though that an impeccable course disclosure custom can't be developed on a lone retransmission by each center point, in light of the way that such a request may disregard to accomplish the objective or find the perfect way. Next, it is inspected that grabbing neighbor learning information with "welcome" get-togethers isn't an insignificant tradition. It is portrayed as the bound position-based organizing traditions that intend to urge the normal skip check (if there ought to be an event of hop by-ricochet asked for and settled piece rate) or to build up the probability of transport[31][32].

- An enamoring open issue for the future researchers is to consider physical-layerbased arrangement and broadcasting it where the centers may change their transmission radii. Anticipated power usage may then be seen as a basic optimality measure. Other than that, the research should address contrasting issues in the arrangement of structure layered traditions. Consider a case such that, if an all the more intense and sensible channel is illustrated, for instance, multi-way obscuring, the surveyed number of packs may encounter the evil impacts of generous change, and the portrayed traditions may require a couple of changes. More sensible 
impediment models can be incorporated, and transport layer traditions in like manner ought to be adjusted.

- A diagram of best in class controlling methods in WSNs is displayed. In any case, it is depicted that the blueprint challenges for coordinating traditions in WSNs is taken after by an expansive investigation of guiding strategies. As a rule, the coordinating frameworks were requested into three classes in perspective of the shrouded framework structure: Ricochet, Different leveled, and Region based guiding.

- One of the essential parts of sensor frameworks is that the plans tend to be incredibly application specific. Thus, a layered view like the one used as a piece of OSI, powers a considerable discipline, and utilization mostly intended for the particular, are quite appealing.

- Correspondence, which is the most imperatively costly piece of the framework, can be dealt with, on an extremely essential level in three particular ways: Center point driven, Data driven, and Position driven. Center driven correspondence is the most noticeable and without a doubt a new perspective, being started now to be used as a piece of the Internet.

- Data driven techniques, on the other hand, tend to give a total course of action, much the same as the case with composed scattering. Yet an organized scattered deal with only a solitary issue, grasps it right.

- Here position-driven procedures have the favored point of view since they don't require particular centers to be locked in with sending, yet use whichever ones give arrangement. A segment of the endeavors exploring the probability of presenting optional code on sensors are Sensor Ware and Maté. The usage of TCL (Tool Command Language) substance and bytecode grants foundation of complex circled counts that can get to all the correspondence and recognizing capacities of each center point. Finally, if sensor frameworks are to be sent in broad sizes, flexibility concerning the amount of center points transforms into a focal factor in picking a corresponding perspective.

\section{WIRELESS SENSOR NETWORK APPLICATIONS}

Sensors focuses are utilized for unfaltering, perceiving occasion ID, occasion divulgence and adjoining control of actuators. The livelihoods of remote sensor are arranged on an extremely essential level joint thriving, military, customary, home and distinctive business spaces [5] [6]

There are numerous utilizations of WSNs in mechanical robotics, activity checking and control, restorative gadget observation and in many different territories. A few of these uses are talked about below as follows,

\subsection{CATASTROPHE RELIEF OPERATION}

In case a domain is represented to have been stricken from some sort of fiasco, for instance, a savage blast, by then the sensor drops fire on the center points from a plane. In any case, the seeing tries and the potential sensor frameworks applications are reviewed, and a blueprint of areas influencing the system of sensor structures is given. Advancement in the progress of sensor, for instance, Miniaturized scale Electro Mechanical Frameworks (MEMS), remote exchanges, introduced structures, passed on orchestrating and remote sensor applications have contributed an expansive change in Remote Sensor System (WSN) starting late. It accessorizes and updates work execution 
both in the field of industry and our dependably life [7]. Remote Sensor System has been everything seen as used as a touch of various locales, especially for affirmation and checking in agribusiness and living space seeing. Condition watching has changed into a basic field of control and accreditation, giving reliable structure and control correspondence with the physical world[22][23].

\subsection{MILITARY APPLICATIONS}

It makes an incredible degree that is essential in military assignments for recognizing and checking pleasant or undermining improvements. The cutting edge perception should be possible through the sensor that focuses to keep a watch on everything if more noticeable mechanical assembly, powers or ammo are required in the battle zone. The substance focuses on atomic and common assaults and thus can be perceived through the sensors focus [8] [9].

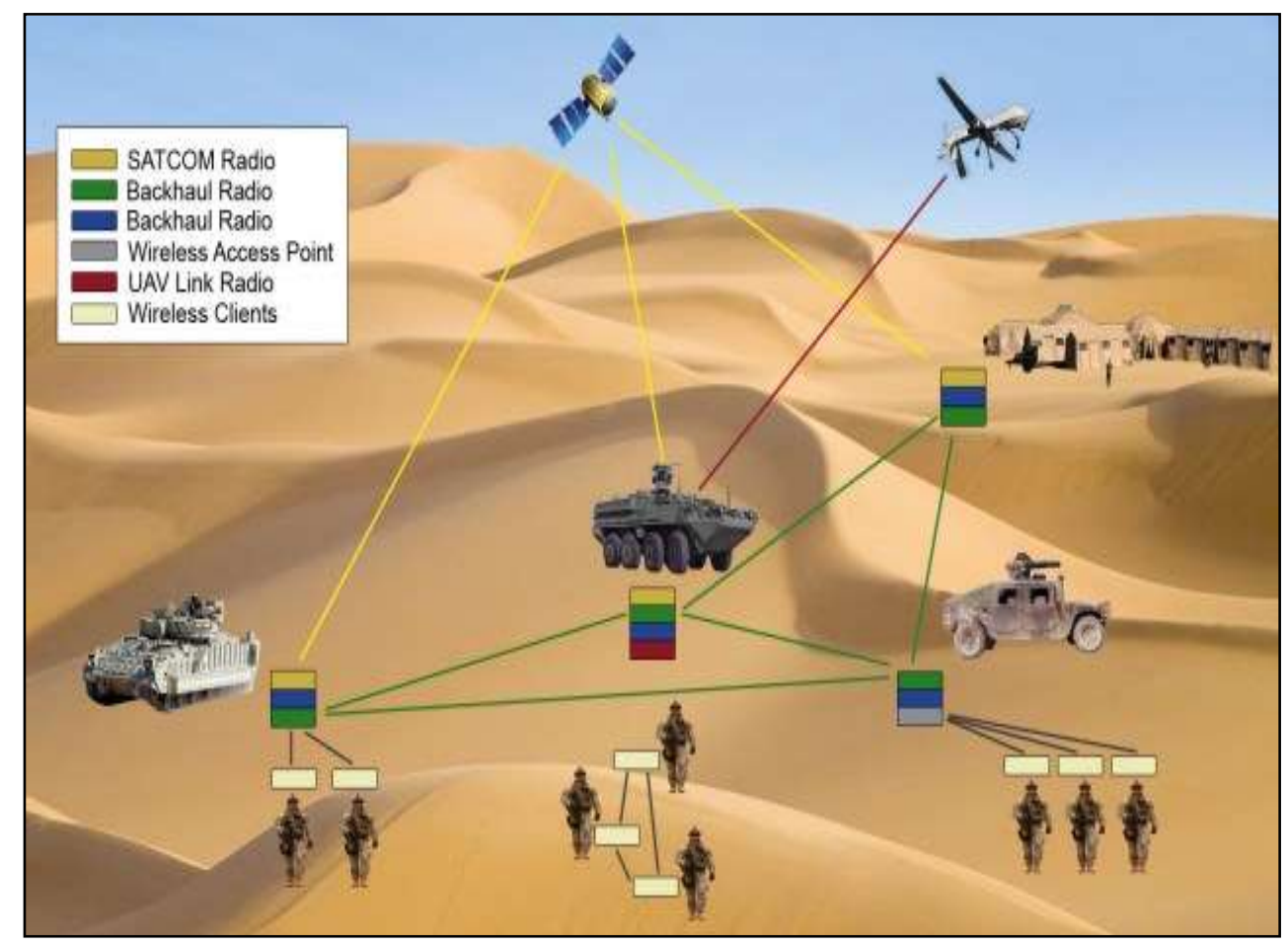

Fig. 3 Military Applications of WSN [12]

\subsection{ECOLOGICAL APPLICATIONS}

Checking of earth soil, climatic changes, water system and accuracy farming should be very much possible with the help of these sensors. They can likewise be utilized for the detection of flame, surge, quakes, and concoction/natural flare-up and so on [7] [10]. 


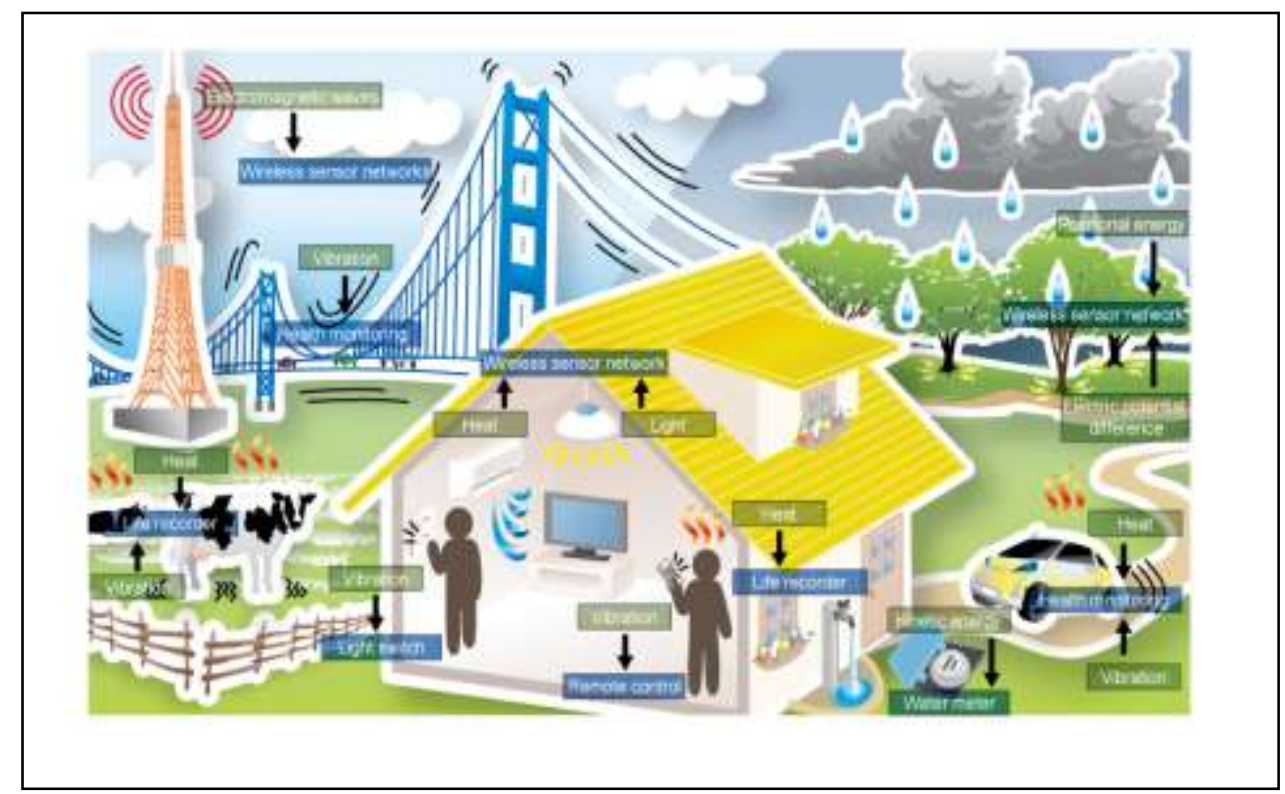

Fig. 4 Ecological Applications [14]

[Fig 4. Source:: https://microcontrollerslab.com/wireless-sensor-networks-wsnapplications/ August 29, 2015 by Microcontrollers Lab ]

\subsection{HOME APPLICATIONS}

Now a several Home Applications were exist in the market. With the help of sensors we can open the door automatically, we can control the Lights, water flow, to close the windows like that so many home applications were there. It all happens just by detecting the motion of body with the help of sensors.

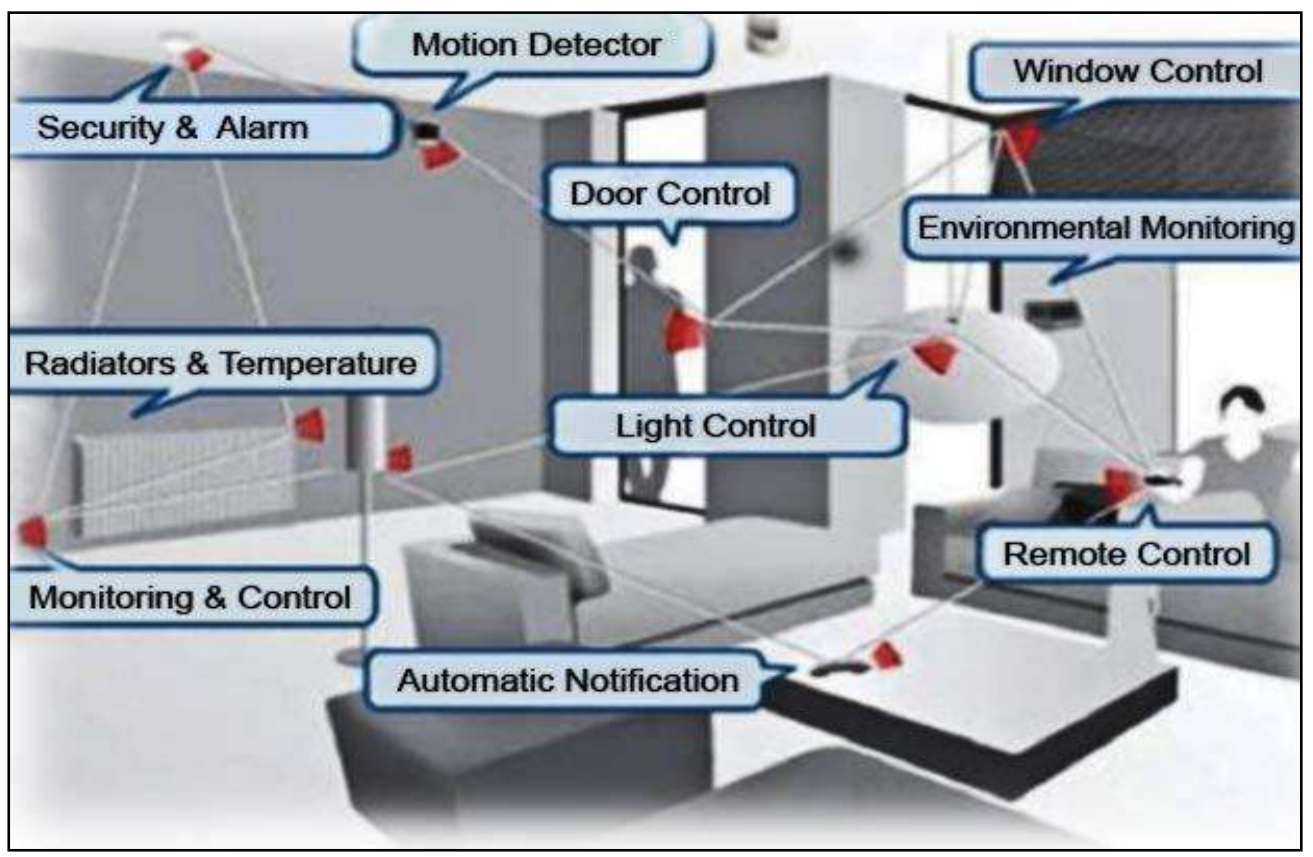

Fig. 5 Home Applications [14]

[Fig 5. Source:: https://microcontrollerslab.com/wireless-sensor-networks-wsnapplications/ August 29, 2015 by Microcontrollers Lab ] 


\section{CONCLUSION}

In this work, an examination on WSN and their advances, guidelines and applications was done. Remote sensor systems include little focuses with recognition, figuring, and remote exchanged limits. Different coordinating, control association, and information scattering conventions have been particularly anticipated WSNs where vital mind is a fundamental game plan issue. Directing conventions in WSNs may contrast contingent upon the application and system design.

At the point when contrasted, the now established MANETs sensors systems have distinctive qualities, and diverse outline and building challenges. One of the primary parts of sensor systems arrangements have a tendency to be exceptionally application specific. Remote specially appointed sensor systems have awesome long haul financial potential, capacity to change our lives, and stance numerous new framework building challenges. Sensor organizers additionally represent various new applied and improvement issues. A few for example are, sending and following principal issues, in those numerous applications, and thus depending on them for the required data. Scope when all is said in done, answers the inquiries regarding nature of administration (reconnaissance) and likewise is a coordinated system for sensor situation that consolidates control administration and adaptation to non-critical failure.

The fundamental topology wanting information gathering remote sensor systems is a crossing tree, since the movement is for the most part as many-to-one streams. A sensor that productively transducers ecological vitality into helpful electrical vitality is a vitality gatherer. With the refinement of vitality collecting methods that can accumulate helpful vitality from vibrations, impacts of radio vitality, and so forth, self-controlled hardware is an undeniable probability. Current security systems in impromptu sensor systems don't ensure dependable and strong system usefulness. Indeed, even with these instruments, the sensor hubs could be made non-operational by pernicious assailants or physical separate of the foundation. Vigor and adaptation to non-critical failure of the sensor arranging topologies comes as a tradeoff with the helplessness of the system topologies to different dangers.

In a steady look for proficient security control and interruption identification frameworks (IDS), a definitive objective in outlining conventions stays less asset utilization while having wide scope and more extensive appropriateness. Remote sensors have turned into a magnificent instrument for military applications including interruption discovery, border observing, data assembling and keen coordination's bolster in an obscure sent zone. Some different applications are: the plan of a sensor-based individual wellbeing screen, area location with sensor systems, and utilizing remote sensor systems to perform development recognition.

\section{REFERENCES}

[1] Faragher, John Mack. "Bungalow and Ranch House: The Architectural Backwash of California." Western Historical Quarterly Vol.32, No.2, 2001, Pp.149-173.

[2] Yick, Jennifier, Bishwanth Mukherjee, and Dipak Ghosal. "Wireless Sensor Network Survey." Wireless Sensor Network Survey. N.p., n.d. Web. 29 Oct. 2016.

[3] Edwin Prem Kumar Gilbert, Baskaran Kaliaperumal, and Elijah Blessing Rajsingh. "Research Issues in Wireless Sensor Network Applications: A Survey." - Volume 2 Number 5 (Sep. 2012). N.p., n.d. Web. 04 Nov. 2016.

[4] "Rover Environmental Monitoring Station (REMS)." NASA. NASA, n.d. Web. 05 Nov. 2016.

[5] V. Ekong and U. Ekong, "A SURVEY OF SECURITY VULNERABILITIES IN WIRELESS SENSOR NETWORKS," Nigerian Journal of Technology, vol. 35, no. 2, p. 392, Apr. 2016.

[6] Gurudatt Kulkarni, Rupali Shelk, Kiran Gaikwad, Vikas Solanke, Sangita Gujar, and Prasad Khatawkar. "WIRELESS SENSOR NETWORK SECURITY THREATS In." IEEE Xplore -. N.p., n.d. Web. 29 Oct. 2016.

[7] Zheng Yue-Feng, Han Jia-Yu, Chen Zhuo-Ran, and Li Zheng. "A Novel Based-node Level Security Strategy in Wireless Sensor Network." 2012 International Conference on Information Management, Innovation Management and Industrial Engineering, n.d. Web. 
[8] Mohamed-Lamine Messai. "Classification of Attacks in Wireless Sensor Networks." International Congress on Telecommunication and Application'14 University of A.MIRA Bejaia, Algeria, 23-24 APRIL 2014, n.d. Web.

[9] Daniel E. Burgner, and Luay A. Wahsheh. "Security of Wireless Sensor Networks." 2011 Eighth International Conference on Information Technology: New Generations, n.d. Web.

[10] Reema Sandhu. "Attacks in Wireless Sensor Networks and Security Measures." IRACST International Journal of Computer Networks and Wireless Communications (IJCNWC), ISSN: 22503501 Vol.6, No 2, Mar-Apr 2016 (n.d.): n. pag. Web.

[11] Kaushal, Kanchan, and Taranvir Kaur. "A Survey on Attacks of WSN and Their Security Mechanisms." International Journal of Computer Applications, Vol.118, No.18, 2015, Pp.1-4.

[12] Swetha. R1, Santhosh Amarnath.V2, Anitha Sofia.V.S3. Wireless Sensor Network: A Survey. nal Journal of Advanced Research in Computer and Communication Engineering, Vol. 7, Issue 11, November 2018.

[13] Satyam Gupta, Wireless Sensor Network: A Survey, Conference Paper, October 2018.

[14] https://www.elprocus.com/architecture-of-wireless-sensor-network-and-applications/.[Last Accessed on 10-05-2019]

[15] https://microcontrollerslab.com/wireless-sensor-networks-wsn-applications/ August 29, 2015 by Microcontrollers Lab. [Last Accessed on 10-05-2019]

[16] Peng Hai-yun, "Energy Consumption of Wireless Sensor Network Research Problem" International Journal of Internet of Things and Big Data. Vol. 1. No. 1. Dec. 2016.GVPress. pp:2936.http://dx.doi.org/10.21742/IJITBD.2016.1.1.04.

[17] Nellore Harika, Muthyala Vamsilatha, Nakka Thirupathi Rao, Debnath Bhattacharyya and Tai-hoon Kim "Performance Analysis and Implementation of Traffic Monitoring System using Wireless Sensor Network for reducing blockage in Traffic on Indian City Roads", Journal of Statistical Computing and Algorithm. Vol. 1. No. 1. Dec. 2017.GVPress. pp:15-26.http://dx.doi.org/10.21742/JSCA.2017.1.1.02.

[18] Yongseong Jo and Kwangteak Kim "Portable wireless smart meter controlled by a smart phone for intelligent energy management", International Journal of Power Devices and Components for Smart Device. Vol. 3. No. 1. Dec. 2016.GVPress. pp:1-8.http://dx.doi.org/10.21742/IJPDCSD.2016.3.1.01

[19] Hyun Joo Park, Seong Cheol Kim and Hye Yun Kim, "Priority Aware Data Collection Mac Protocol in Wireless Sensor Networks", International Journal of Advanced Science and Technology, NADIA, ISSN: 2005-4238 (Print); 2207-6

[20] Yukun Yao, Yaorui Liu and Jikai Yang, "An Efficient RPL Protocol in Wireless Sensor Networks Based on Energy Balance", International Journal of Advanced Science and Technology, SERSC Australia, ISSN: 2005-4238 (Print); 2207-6360 (Online), vol. 98, January (2017), pp. 33-44, http://dx.doi.org/10.14257/ijast.2017.98.03.

[21] N. Thirupathi Rao, U. Nanaji, Ch. Raj Kumar, Debnath Bhattacharyya and Hye-jin Kim, "Performance Analysis of Wireless Sensor Network with IEEE 802.15.4 Protocol", International Journal of Control and Automation, SERSC Australia, ISSN: 2005-4297 (Print); 2207-6387 (Online), vol. 9, no. 12, December (2016), pp.383-394, http://dx.doi.org/10.14257/ijca.2016.9.12.32.

[22] Woosik Lee, Teuk-Seob Song and Jong-Hoon Youn, "Asymmetric Neighbor Discovery Protocol for Wireless Sensor Networks using Block Design", International Journal of Control and Automation, SERSC Australia, ISSN: 2005-4297 (Print); 2207-6387 (Online), vol. 10, no. 1, January (2017), pp.387396, http://dx.doi.org/10.14257/ijca.2017.10.1.35.

[23] Xing-Po Ma, Guo Song, Lei Li, Yan-Ge Sun and Jun-Bin Liang, "Comparing and Analysis on Adaptive Information-brokerage Schemes in Wireless Sensor Networks", International Journal of Grid and Distributed Computing, SERSC Australia, ISSN: 2005-4262 (Print); 2207-6379 (Online), vol.10, no.5, May (2017), pp. 25-40, http://dx.doi.org/10.14257/ijgdc.2017.10.5.03.

[24] Ms. L. S. Sindhuja and Dr. G. Padmavathy, "Clone Detection Using Enhanced EDD (EEDD) with Danger Theory in Mobile Wireless Sensor Network", International Journal of Security and Its Applications, SERSC Australia, ISSN: 1738-9976 (Print); 2207-9629 (Online), vol.9, no.4, April (2015), pp. 185-202, http://dx.doi.org/10.14257/ijsia.2015.9.4.18.

[25] Wengen Gao, Qigong Chen, Ming Jiang, Yunfei Li, Shinong Wang, "The Optimization of Genetic Algorithm in Wireless Sensor Network Coverage", International Journal of Signal Processing, Image Processing and Pattern Recognition, SERSC Australia, ISSN: 2005-4254 (Print); 2207-970X (Online), vol.8, no.1, January (2015), pp. 255-264, http://dx.doi.org/10.14257/ijsip.2015.8.1.22.

[26] Bin Zhu, Jincheng Li, Wenshan Hu and Xingran Gao, "Review of Magnetic Coupling Resonance Wireless Energy Transmission", International Journal of u - and e - Service, Science and Technology, SERSC Australia, ISSN: 2005-4246 (Print); 2207-9718 (Online), vol. 8, no.3, March (2015), pp. 257272, http://dx.doi.org/10.14257/ijunnesst.2015.8.3.25.

[27] Lovepreet Kaur and Jyoteesh Malhotra, "Comparison of Wise Route and Flooding Network Type of Convergecast Routing in Wireless Sensor Network", International Journal of Sensor and Its Applications for Control Systems, SERSC Australia, ISSN: 2287-8467 (Print); 2207-6344 (Online), vol.3, no.2, November (2015), pp. 1-10, http://dx.doi.org/10.14257/ijsacs.2015.3.2.01. 
[28] Zhang, Wei; Xiong, Naixue; Yang, Laurence T.; Jia, Gangmin; Zhang, Jilin.: BCHED - Energy Balanced Sub-Round Local Topology Management for Wireless Sensor Network. JOURNAL OF INTERNET TECHNOLOGY, 2012, 13(3): 385-394.

[29] Yuyu Yin, Song Aihua, Gao Min, Yueshen Xu, Wang Shuoping:QoS Prediction for Web Service Recommendation with Network Location-Aware Neighbor Selection. International Journal of Software Engineering and Knowledge Engineering 26(4): 611-632 (2016).

[30] Jin Wang, Yu Gao, Wei Liu, Arun Kumar Sangaiah, Hye-Jin Kim, Energy Efficient Routing Algorithm with Mobile Sink Support for Wireless Sensor Networks, Sensors, 2019, 19(7), 1494. doi:10.3390/s19071494.

[31] Jin Wang, Yu Gao, Wei Liu, Arun Kumar Sangaiah, Hye-Jin Kim, An Improved Routing Schema with Special Clustering using PSO Algorithm for Heterogeneous Wireless Sensor Network, Sensors, vol.19, no.3, Feb. 2019. 10.3390/s19030671

[32] Jin Wang, Yu Gao, Wei Liu, Wenbing Wu, Se-Jung Lim, An Asynchronous Clustering and Mobile Data Gathering Schema based on Timer Mechanism in Wireless Sensor Networks, Computers, Materials \& Continua, Vol.58, No.3, pp.711-725, 2019. 